\title{
The Anti-Inflammatory Effect of Zhibaidihuang Decoction on Recurrent Oral Ulcer with Sirt1 as the Key Regulatory Target
}

\author{
Yajie Shao, ${ }^{1}$ Bin Ding $\mathbb{D}^{2},{ }^{2}$ Jinjun Ji, ${ }^{1}$ Shanshan Lei, ${ }^{1}$ Yu Dong, ${ }^{3}$ Yongsheng Fan $\mathbb{D}^{4}{ }^{4}$ \\ Weihong Ge, ${ }^{5}$ and $\mathrm{Li} X u \mathbb{D}^{1}$ \\ ${ }^{1}$ College of Basic Medicine, Zhejiang Chinese Medical University, Hangzhou, Zhejiang, China \\ ${ }^{2}$ College of Life Science, Zhejiang Chinese Medical University, Hangzhou, Zhejiang, China \\ ${ }^{3}$ Zhejiang Academy of Traditional Chinese Medicine, Hangzhou, Zhejiang, China \\ ${ }^{4}$ First Clinical Medical College, Zhejiang Chinese Medical University, Hangzhou, Zhejiang, China \\ ${ }^{5}$ College of Pharmaceutical Science, Zhejiang Chinese Medical University, Hangzhou, Zhejiang, China \\ Correspondence should be addressed to Li Xu; xulihhb@163.com
}

Received 30 September 2020; Revised 10 April 2021; Accepted 19 April 2021; Published 3 May 2021

Academic Editor: Jing Zhao

Copyright (C) 2021 Yajie Shao et al. This is an open access article distributed under the Creative Commons Attribution License, which permits unrestricted use, distribution, and reproduction in any medium, provided the original work is properly cited.

\begin{abstract}
The syndrome of ROU is generally manifested as obvious pain, redness, and swelling of local ulceration area, accompanied by flushed face, red eyes, sore throat, and swollen gums. Traditional Chinese medicine (TCM) doctors believe that "yin deficiency" is one causative factor of ROU. Zhibaidihuang decoction (ZBDHD) is a prescriptively developed receipt, where Anemarrhena asphodeloides and Phellodendri amurensis Cortex are added in the original Liuweidihuang decoction. It is generally used for "yin deficiency" treatment. It can effectively reduce the recurrence of oral ulcers and release the severity of the disease. However, the mechanism of this activity remains to be elucidated. In this study, we found that ZBDHD has a certain therapeutic effect on the pathological changes of oral mucosa. Furthermore, the results of serum metabolomics showed ZBDHD influenced the synthesis and metabolism of certain fatty acids. The results of western blot, immunochemical, and immunofluorescence staining indicate that ZBDHD could increase the expression of Sirt1 and Foxp3 and suppress the expression and acetylation of NF- $\kappa \mathrm{B}$ in oral mucosa cells. By screening active ingredients in ZBDHD, we found berberine, as well as other compounds, presenting high fitness of the Sirt1 reactive centre. Therefore, it is possible that ZBDHD can regulate the Sirt1-NF- $\kappa$ B pathway to improve fatty acids metabolism in the body, thereby achieving the effect of treating ROU.
\end{abstract}

\section{Introduction}

Recurrent oral ulcer (ROU) is one of the most common oral mucosal diseases and its incidence rate is 30\% [1]. Come so far, the mechanism is still unclear; most studies indicate that ROU is associated with immune inflammation [2]. It was considered that the ROU included five stages including initiation, primary damage response, expanded damage response, ulcer formation, and repair, while inflammatory response was throughout the process. The clinical research showed that $\mathrm{CD} 4^{+}, \mathrm{CD} 25^{+}$, and Foxp $3^{+}$were significantly reduced [3]. On the contrary, IL- 6 and TNF- $\alpha$ concentration was significantly increased in peripheral blood of recurrent ulcers patients [4]. Animal experiments showed that the expression of NF- $\kappa \mathrm{B}$ p65 [5] and TNF- $\alpha$ significantly increased in recurrent oral ulcer model rats [6]. These previous reports demonstrated that ROU is related to immune response and inflammatory response.

The Chinese medicine theory mentions that the unevenness of "yin" (cold) and "yang" (hot, fire) in the human body could result in diseases. TCM doctors considered that excess "yang" (fire) is an important factor in causing ROU in clinic [7]. The excess fire could be due to "yinxu," which means "yin deficiency". Fuzi (Radix Praeparata Aconitum carmichaelii Debx), Ganjiang (Rhizoma Zingiber officinale), and Rougui (Cortex Cinnamomum cassia) decoction (AZC) is one of the prescriptions for hyperthermia, used to treat "cold" syndrome. However, overdose of AZC could break 
the balance between "yin" and "yang," which produces heat and inflammation [8]. Previous work indicated that AZC could induce excess of "fire" to cause oral ulcer $[9,10]$.

Zhibaidihuang decoction (ZBDHD), a traditional Chinese herbal receipt, is usually used to cure ROU and hyperactivity of "fire" due by "yin" deficiency in clinic $[11,12]$. The receipt was composed of eight herbs including Zhimu (Rhizoma Anemarrhena asphodeloides), Huangbai (Phellodendri amurensis Cortex), Shudihuang (Radix Rehmanniae Preparata), Shanzhuyu (Fructus Cornus officinalis), Shanyao (Radix Dioscorea opposita), Fuling (Sclerotium of Poria cocos), Mudanpi (Cortex Paeonia suffruticosa), and Zexie (Rhizoma Alisma orientale). In this prescription, Fuling, Mudanpi, and Zexie have capability of eliminating excess "yang" (fire). And Huangbai and Zhimu show activity on enhancing the efficiency. The active components in this receipt are very complex. This is due to the complex mechanisms of ZBDHD treatment. It was identified with modern biological method that AZC can influence the AMPK/Sirt1 pathway [10], as well as expression of NF- $\kappa \mathrm{B}$, one of inflammatory response cytokines [13]. Previous studies proved that Sirt1 was correlated to cell metabolism $[14,15]$. And Sirt1 is the key cytokine, which regulates the transcription of $\mathrm{NF}-\kappa \mathrm{B}[16,17]$. Therefore, in this research, we evaluated the regulation mechanism of ZBDHD treatment on oral mucosa through Sirt1/NF- $\kappa$ B pathway with a AZC treated ROU rat model.

\section{Materials}

2.1. Materials and Regents. Freund's complete adjuvant (8642852) was purchased from MP Biomedicals (California, USA). The specific antibodies against Sirt1 (sc-74465), NF$\kappa \mathrm{B}$ p65 (sc-372), Stat3 (sc-482), Foxp3 (sc-28705), acetyl lysine (sc-284922), and TNF- $\alpha$ (sc-4261) were all purchased from Santa Cruz Biotechnology (Texas, USA). The secondary antibodies, Goat anti Rabbit IRDYE @ 800 CW (C10324-01), and Goat anti Mouse IRDYE @ 680 (C4031202) were all purchased from LICOR (Nebraska, USA). Fetal bovine serum and RPMI1640 cell culture medium were obtained from GENOM (Hangzhou, China). The primers used in this study were synthesized by Bioengineering (Shanghai, China).

2.2. Animals. Fifty healthy $(180 \sim 200 \mathrm{~g})$ female SD rats were purchased from Shanghai SLAC Lab. Animal Ltd. (Shanghai, China) and housed in laboratory animal research centre of Zhejiang Chinese Medical university. All animal experiments have passed the ethics committee with accepted $\mathrm{Nr}$. ZSLL-2016-116.

2.3. Decoction Preparation. All of the herbs were purchased from Zhejiang Province Famous Doctor Pavilion and Zhejiang Chinese Medical University Medical Slicing Factory. The preparation of AZC decoction refers to our previous publication [8]. ZBDHD preparation was also described previously [13] and was diluted to specific concentration for experiments. And the decoction concentration in this work presented as milli gram herbal mixture per milli liter.

2.4. Animal Treatment. In this experiment, six rats (control group, Ct), were given distilled water. For model preparation, the oral mucosa from twenty healthy $\mathrm{SD}$ rats was mixed with complete Freund's adjuvant $(1: 1 v / v)$ and sterile-filtrated with a sterile filter $(0.22 \mu \mathrm{m}$ diameter $)$. Twenty-four rats were subcutaneously injected $0.1 \mathrm{~mL}$ this mucosa emulsion on both sides of back once a week. And rats were internally orally injected $0.1 \mathrm{~mL}$ mucosa emulsion, also. Simultaneously, rats were given $4 \mathrm{~mL} 1 \mathrm{~g} / \mathrm{mL}$ AZC by intragastric administration (i.g.) every day for 4 weeks. For ZBDHD protective efficiency evaluation, these twenty-four rats were separated into four groups. One group (model group, AZC) was given $4 \mathrm{~mL} /$ day distilled water by intragastric administration (i.g.). Simultaneously, the other three groups were treated with $4 \mathrm{~mL} /$ day high dose $(2 \mathrm{~g} / \mathrm{mL})$, middle dose $(1 \mathrm{~g} / \mathrm{mL})$, and low dose $(0.5 \mathrm{~g} / \mathrm{mL})$ ZBDHD by i.g. for a week, respectively, which were marked as ZBDHD-H, ZBDHD-M, and ZBDHD-L.

2.5. Histological Study. The $10 \%$ formalin solution fixed oral mucosa (of each rat in different groups) was embedded in paraffin which was cut and stained with Hematoxylin \& Eosin (H\&E) stain for electron microscope (Carl Zeiss, Germany) observation.

2.6. Immunohistochemistry and Immunofluorescence Image The paraffin-embedded sample was cut into thin sections (4$5 \mu \mathrm{m}$ ) and sealed in $3 \% \mathrm{H}_{2} \mathrm{O}_{2}$ at room temperature to inactivating enzymes. Then, the sections were boiled in $10 \mathrm{mM}$ sodium citrate buffer $(\mathrm{pH}$ 6.0) for $10 \mathrm{~min}$ and cooled at room temperature. For immunohistochemistry analysis, the sections were hybridized with primary antibodies against acetyl lysine $(1: 200)$, Sirt $1(1: 200)$, NF- $\kappa$ B $(1: 200)$, and TNF- $\alpha(1: 200)$, respectively, overnight at $4^{\circ} \mathrm{C}$. The primary antibodies were detected with fluorescence labelled corresponding secondary antibody. Simultaneously, API staining was performed to stain the nuclei. More details were described in previous studies $[9,18]$.

\subsection{Serum Preparation and Metabolites Analysis}

2.7.1. Serum Preparation. The blood samples were stated at $37^{\circ} \mathrm{C}$ for one hour and centrifugated at $4000 \mathrm{rpm}$ for $10 \mathrm{~min}$. $150 \mu \mathrm{L}$ serum was pipetted and vortex mixed with $450 \mu \mathrm{L}$ acetonitrile to precipitated proteins. The supernatant was diluted $1: 1$ with deionized water and filtered with a $0.22 \mu \mathrm{m}$ nylon filter.

2.7.2. HPLC-QTOF/MS Analysis. $5 \mu \mathrm{L}$ filtered serum samples were loaded into a Waters Acquity UPLC C18 column $(1.8 \mu \mathrm{m}, \quad 2.1 \times 50 \mathrm{~mm})$, with Agilent $1260 \mathrm{HPLC} / 6520$ QTOF-MS instrument (Agilent, Milford, MA, USA). The mobile phase consisted of $95 \%$ solvent A $(0.1 \%(v / v)$ formic acid) and $5 \%$ solvent B $(0.1 \%(v / v)$ formic acid acetonitrile 
solution). The optima gradient eluate programme was as follows: $0-4 \mathrm{~min}, 95 \% \mathrm{~A}$; $4-17 \mathrm{~min}, 90 \% \mathrm{~A}$ to $55 \% \mathrm{~A}$; $17-22 \mathrm{~min}, 55 \% \mathrm{~A}$ to $5 \% \mathrm{~A}$. The parameters of electrospray ionization $(\mathrm{ESI}+)$ mass spectrum were drying gas $\left(\mathrm{N}_{2}\right)$ flow rate, $10 \mathrm{~L} / \mathrm{min}$; gas temperature, $350^{\circ} \mathrm{C}$; scan range, $\mathrm{m} / \mathrm{z}$ 50 1000; capillary voltage, $4000 \mathrm{~V}$; fragmentation voltage, $180 \mathrm{~V}$; cone voltage, $60 \mathrm{~V}$; atomizing air pressure, $310 \mathrm{kPa}$. The acquisition rate was 2 spectra/s.

2.8. Western Blot. The expressions of signalling molecules, Sirt1, NF- $\kappa$ B p65, Stat3, and Foxp3 in oral mucosa cell from different group rats were comparatively identified with western blot. The oral mucosa was sheared in lysate buffer by tissue homogenizer, and cell debris was discharged by centrifugation. The protein concentration in cell lysate was quantified with Bradford. 20-30 $\mu$ g proteins were mixed with loading buffer and separated with an SDS-PAGE gel. For western blot analysis, the proteins in gel were electrically transferred on a nitrocellulose filter membrane at $80 \mathrm{~V}$ for 2 hours in a transfer buffer system $(3.03 \mathrm{~g}$ Tris $+14.4 \mathrm{~g}$ glycine $+200 \mathrm{~mL}$ methanol in $\mathrm{ddH}_{2} \mathrm{O}$ to a final volume of $1000 \mathrm{~mL}$ ). Before hybridization, the membrane was cut according to the mass of detected proteins and then incubated with antibodies specific for $\beta$-actin, Sirt1, NF- $\kappa \mathrm{B}$ p65, Stat 3 , or Foxp 3 separately at $4^{\circ} \mathrm{C}$ overnight. On the next day, membranes were washed and incubated with the respect secondary antibody for 2 hours. The blots were exposed and scanned using an Odyssey Infrared Imaging system (LICOR Biosciences), and the blots were quantified using the Odyssey Infrared Scanning system software.

2.9. Identification of Sirt1 Regulator(s) in ZBDHD. All chemical compounds in ZBDHD were first selected from the TCMSP database (http://lsp.nwu.edu.cn/tcmsp.php) [19]. Three in silico ADME models, OB (oral bioavailability, threshold $\geq 30 \%$ ), DL (drug-likeness, threshold $\geq 30 \%$ ), and Caco-2 (Caco-2 permeability, threshold $\geq 30 \%$ ), were used, respectively. The inhibitory affinity of eight major components with human Sirt1 was validated by molecular docking using AutoDock4.2.0 software [20].

2.10. Statistical Analysis. All the data in this study were expressed as mean \pm standard deviation (SD). And $t$-test was used to determine the differences between control and treatment groups with SPSS 15.0. The statistically significant difference was considered at level $P<0.05$.

\section{Results}

3.1. Pathological Changes of Oral Mucosa Induced by AZC and Proactive Effect of $Z B D H D$. In 4 weeks, the oral mucosa of the model group exhibited irritation and inflammation, which is shown in Figure 1(a) and indicated by a black arrow. The aberrant hyperplastic epithelium and infiltrating of inflammatory cells could be observed in $\mathrm{H} \& \mathrm{E}$ strained oral mucosa of AZC model rats (Figure 1(c)), but not in control group rats (Figure 1(b)). In comparison with that of rats in AZC model group, oral mucosa of ZBDHD (high dose and middle dose) (Figures $1(\mathrm{~d})$ and $1(\mathrm{e})$ ) treated rats showed clear cell structure and improved of inflammation, obviously. However, no positive effects could be observed in ZBDHD (low dose) group (Figure 1(f)). The changes of body weights during the last 7 days treatment were monitored (Table 1). Although those of the ZBDHD treated rats were larger than those of model rats, no significant differences were observed.

3.2. ZBDHD Influences the Metabolites Variation. As a part of system biology, metabolomics is a new discipline which can demonstrate the curative effect of TCM therapy. In this study, metabolic profiles of serum samples from rats in control and ZBDHD-H (Figure 2(a)) groups were analyzed by HPLC-QTOF/MS. The data were discriminated with the principal component analysis (PCA) (Figure 2(b)), partial least squares discriminant analysis (PLS-DA) (Figure 2(c)), and the orthogonal partial least squares-discriminate analysis (OPLS-DA) (Figure 2(d)). The metabolites were considered as potential biomarkers responsible for the metabolic profile, when $P$ value of $t$-test $<0.05$ and VIP $>1$. In comparison with those of control group, phytosphingosine, palmitic amide, and MG $(0: 0 / 16: 0 / 0: 0)$ in serum of middle dose ZBDHD treated rats were significantly decreased. On the contrary, $\alpha$-Linolenic Acid, LysoPC (20: 4 (5Z, 8Z, 11Z, 14Z), LysoPC (20:3 (5Z, 8Z, $11 Z)$, and LysoPE $(0: 0 / 20: 0)$ were significantly increased (Table 2). Screening KEGG database [9], these metabolites were related to fatty acid biosynthesis, linoleic acid, and glycerophospholipid metabolism (Table S1). And Sirt1 could be one of the key regulatory cytokines.

\subsection{The Effect of ZBDHD on Sirt1/NF- $\kappa B$ Pathway in Oral} Mucosa. Sirt1 is a protein deacetylase that interferes with the NF- $\kappa \mathrm{B}$ signaling pathway, which regulates cell anti-inflammatory function. Therefore, the expression of cytokines, Sirt $1, N F-\kappa B$, and TNF- $\alpha$, in oral mucosa of rats in different groups, was immunohistochemically imaged in brown (Figure 3). In the cell of model rats, the expressed Sirt1 was obviously less than those of control rats. On the contrary, the expressed NF- $\kappa \mathrm{B}$ and TNF- $\alpha$ were more than those of control group. The expression of Sirt1, NF- $\kappa \mathrm{B}$, and TNF- $\alpha$ in oral mucosa cells of rat was ameliorated by ZBDHD treatment. Simultaneously, the location of Sirt1 (red stained) and deacetylated NF- $\kappa \mathrm{B}$ (green stained) in cells was studied with immunofluorescence detection (Figure 4 ). The yellow signal could be obtained in merged photos. In comparison, in the model group (Figure 4 Merge) more yellow signal could be observed than in the other two groups. To confirm this result, the expression of Sirt1 and NF- $\kappa \mathrm{B}$ in cells was studied by western blot, as well as Foxp3 and Stat3. The high dose ZBDHD treatment $(2 \mathrm{~g} / \mathrm{mL})$ could inhibit the expression of $\mathrm{NF}-\kappa \mathrm{B}$ and stimulate the expression of Sirt1, significantly (Figure 5). In addition, the expression of Foxp3 was also influenced by the treatment in a dose-dependent manner. However, the expression of Stat 3 was stimulated by ZBDHD but not significantly. 


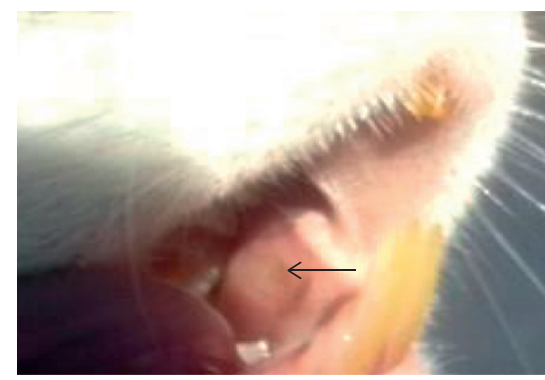

(a)

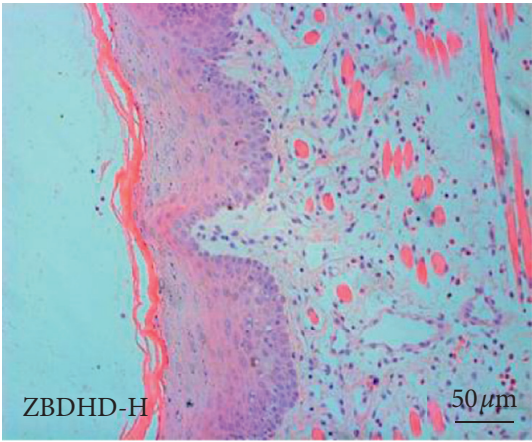

(d)

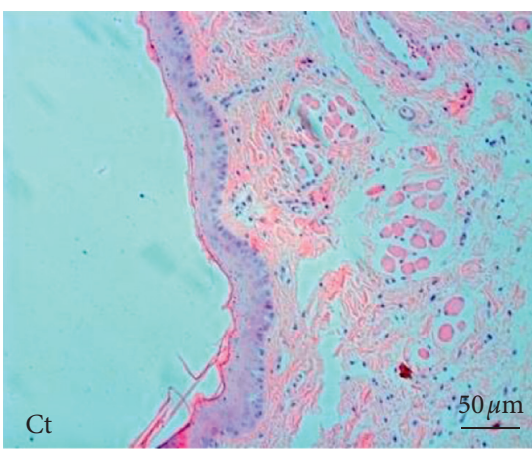

(b)

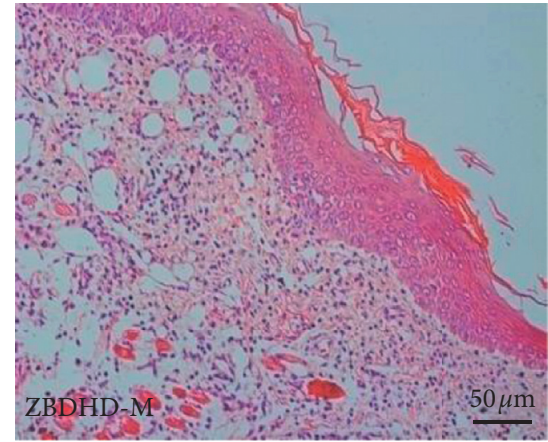

(e)

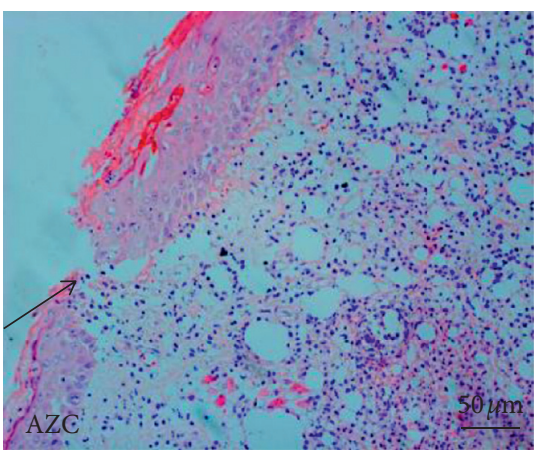

(c)

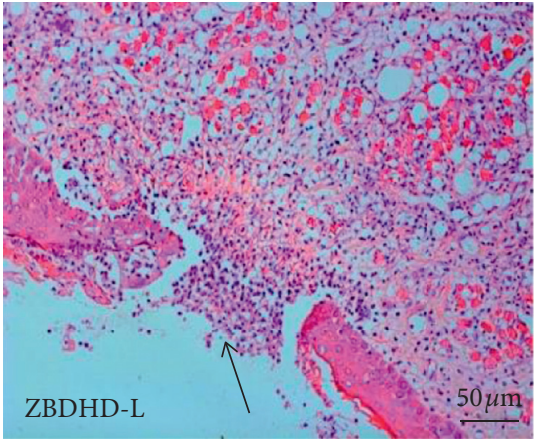

(f)

FIGURE 1: AZC model and protective effect on oral mucosa of ZBDHD treatments. (a) Ulcer (indicated by the black arrow) in oral mucosa of AZC model rat. (b-f) Histopathologic study of oral mucosa stained by hematoxylin and eosin (H\&E) (x200); Ct, AZC, ZBDHD-H, ZBDHD-M, and ZBDHD-L indicated control, AZC model, high-dose ZBDHD treatment (2 g/mL), middle-dose ZBDHD treatment (1 g/ $\mathrm{mL})$, and low-dose ZBDHD treatment $(0.5 \mathrm{~g} / \mathrm{mL})$, respectively.

TABLE 1: The body weight monitor in the last 7 days.

\begin{tabular}{lccc}
\hline Group & Initial weight $(\mathrm{g})$ & Final weight $(\mathrm{g})$ & Increase $(\mathrm{g})$ \\
\hline Control group & $255.83 \pm 12.01$ & $263.83 \pm 11.20$ & $8.00 \pm 5.40$ \\
AZC group & $236.83 \pm 9.33$ & $241.17 \pm 7.91$ & $4.33 \pm 2.80$ \\
ZBDHD-H & $239.33 \pm 7.12$ & $246.67 \pm 9.79$ & $7.33 \pm 3.44$ \\
ZBDHD-M & $233.17 \pm 12.46$ & $242.00 \pm 16.19$ & $8.83 \pm 5.60$ \\
ZBDHD-L & $228.17 \pm 8.52$ & $235.00 \pm 8.88$ & $6.83 \pm 3.66$ \\
\hline
\end{tabular}

Note: the data are expressed as mean $\pm \mathrm{SD}(n=6)$.

The position of cell nucleus, acetyl lysine, and NF- $\kappa \mathrm{B}$ was, respectively, hybridized with blue, red, and green fluorescein labeled specific antibodies. The yellow spot, in merged image, indicated the co-location of acetyl lysine and NF- $\kappa \mathrm{B}$ (red arrow marked).

3.4. Screening Active Ingredients in ZBDHD Which Docked the Reactive Centre in Sirt1. Bioinformatic technology was engaged in identifying the active substances in ZBDHD. Based on TCMSP database (http://lsp.nwu.edu.cn/tcmsp.php), there were 729 kinds of known ingredients, including 81 kinds in Rhizoma Anemarrhena asphodeloides, 141 kinds in Phellodendri amurensis Cortex, 76 in Radix Rehmanniae preparata, 226 in Fructus Cornus officinalis, 71 in Radix Dioscorea opposita, 34 in Sclerotium of Poria cocos, 55 in Cortex Paeonia suffruticosa, and 46 in Rhizoma Alisma orientale in eight herbs of ZBDHD. In silico ADME molecular docking model $(\mathrm{OB} \geq 30 \%, \mathrm{DL} \geq 0.18$ and Caco$2 \geq 0.4$ ) was utilized to virtually screen these ingredients, in which 78 compounds represent the potential docking properties on Sirt1 (Table S2). The interaction specification was evaluated by affinity score. And eight of the most sensitive candidates are shown in Table 3, according to their affinity score.

Berberine is one well-known component in Phellodendri amurensis Cortex. We hypothesized that berberine (Figure 6(a)) can dock in the reactive centre of Sirt1. A docking model was generated with the well-known Sirt1 inhibitor, $1 \mathrm{NS}$ (4-(4-[2-[[methylsulfonyl] amino] ethyl] piperidin-1-yl)thieno (3, 2-d)pyrimidine-6-carboxamide) (Figure 6(b)). The superimposition of $1 \mathrm{NS}$ and berberine is shown in Figure 6(c). The active centre parameters of berberine in Sirt1 were $X=5.641 ; Y=41.417 ; Z=-1.615$, which was calculated with the Autodock 4.2.0 software. The $3 \mathrm{D}$ structure of Sirt1-berberine complex was figured out by PyMol software (Figure 6(d)). The binding energy of Sirt1 and berberine was $-9.9 \mathrm{kcal} / \mathrm{mol}$, which indicated a good interaction result. From the complex, amino acid residues of PHE-273 in Sirt1 and benzene in berberine could form the $\pi-\pi$ conjugated.

\section{Discussion and Conclusion}

Herbal medicines have been used to treat various diseases for many centuries, in Chinese society. Zhibaidihuang 


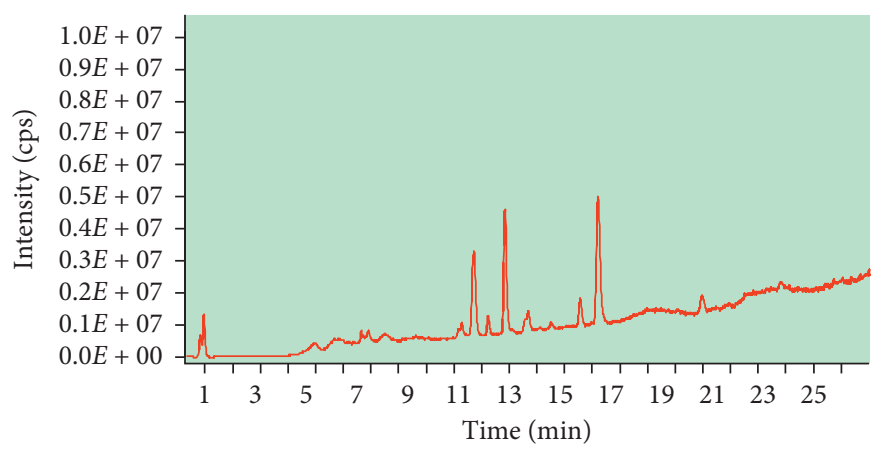

(a)

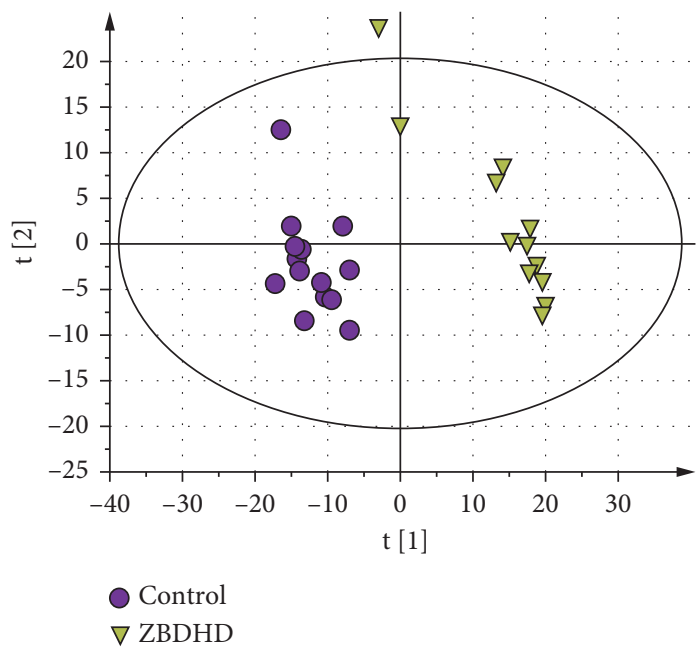

(c)

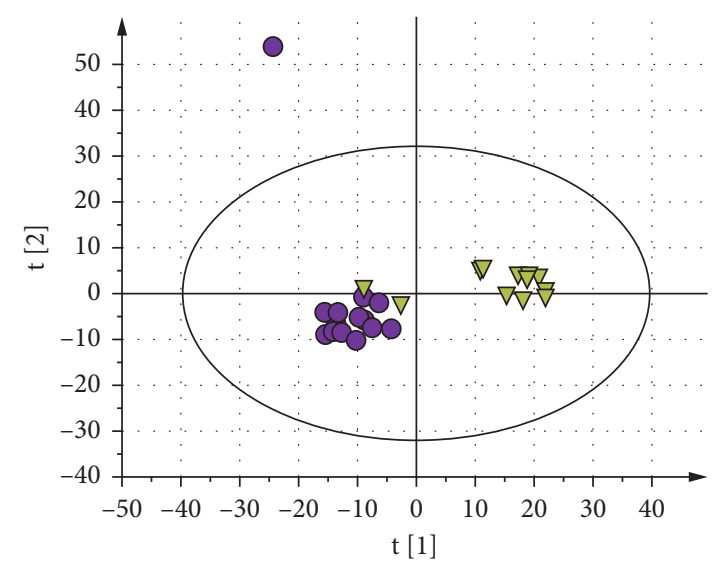

Control

$\nabla$ ZBDHD

(b)

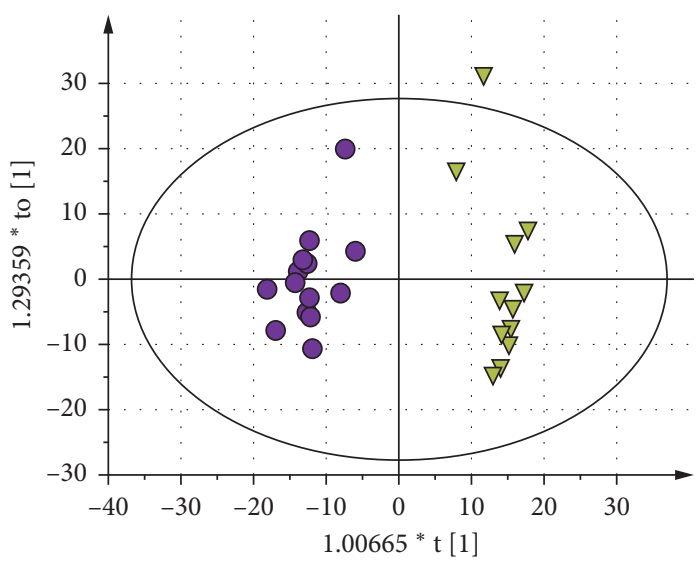

- Control

$\nabla$ ZBDHD

(d)

FIgURE 2: HPLC-QTOF/MS analysis of the serum from the ZBDHD-H treated rat. (a) HPLC-based analysis of serum. (b) PCA score plot of serum samples between the rats in ZBDHD and those in control group (R2X=0.42, Q2 =0.0913); (c) PLS-DA score plot between the two groups $(\mathrm{R} 2 \mathrm{X}=0.281, \mathrm{R} 2 \mathrm{Y}=0.953, \mathrm{Q} 2=0.777)$; and $(\mathrm{d})$ OPLS-DA score plot between the two groups $(\mathrm{R} 2 \mathrm{X}=0.281, \mathrm{R} 2 \mathrm{Y}=0.953, \mathrm{Q} 2=0.772)$ : control group and ZBDHD group.

TABLE 2: Differences metabolites between control and ZBDHD group in OPLS-DA analysis.

\begin{tabular}{lccccc}
\hline Name & VIP & RT & Mass & $T$ test & Fold change $(\mathrm{D} / \mathrm{C})$ \\
\hline Phytosphingosine & 1.593 & 6.686 & 317.2931 & 0.034 & -1.159 \\
Palmitic amide & 1.857 & 7.096 & 255.2558 & 0.012 & -2.183 \\
$\alpha$-Linolenic acid & 1.785 & 7.306 & 278.2241 & 0.016 & 0.923 \\
LysoPC $(20: 4(5 Z, 8 Z, 11 Z, 14 Z))$ & 1.813 & 10.710 & 543.3330 & 0.014 & 0.650 \\
LysoPC $(20: 3(5 Z, 8 Z, 11 Z))$ & 1.570 & 11.839 & 545.3474 & 0.037 & 0.249 \\
LysoPE $(0: 0 / 20: 0)$ & 2.086 & 13.097 & 509.3462 & 0.004 & 1.406 \\
MG $(0: 0 / 16: 0 / 0: 0)$ & 1.522 & 18.784 & 330.2751 & 0.044 & -1.194 \\
\hline
\end{tabular}

Note: fold change (D/C) is the logarithm of the ratio of the mean values of $D$ to $C$ (base 2). The positive sign indicates that $D$ is increased relative to $C$ and the negative sign indicates descent.

decoction (ZBDHD), a poly herbal formula, has been used to treat syndrome of hyperactivity of "yang" (fire) due to "yin" deficiency for thousands of years [21]. Recurrent oral ulcers are a typical symptom of hyperactivity of "fire" due to "yin" deficiency [22]. In modern medicine, oral ulcer is a kind of inflammatory diseases, which occurs due to a damage in epithelium induced by many certain factors, such as hormonal changes [23], lack of essential vitamins [24], and 

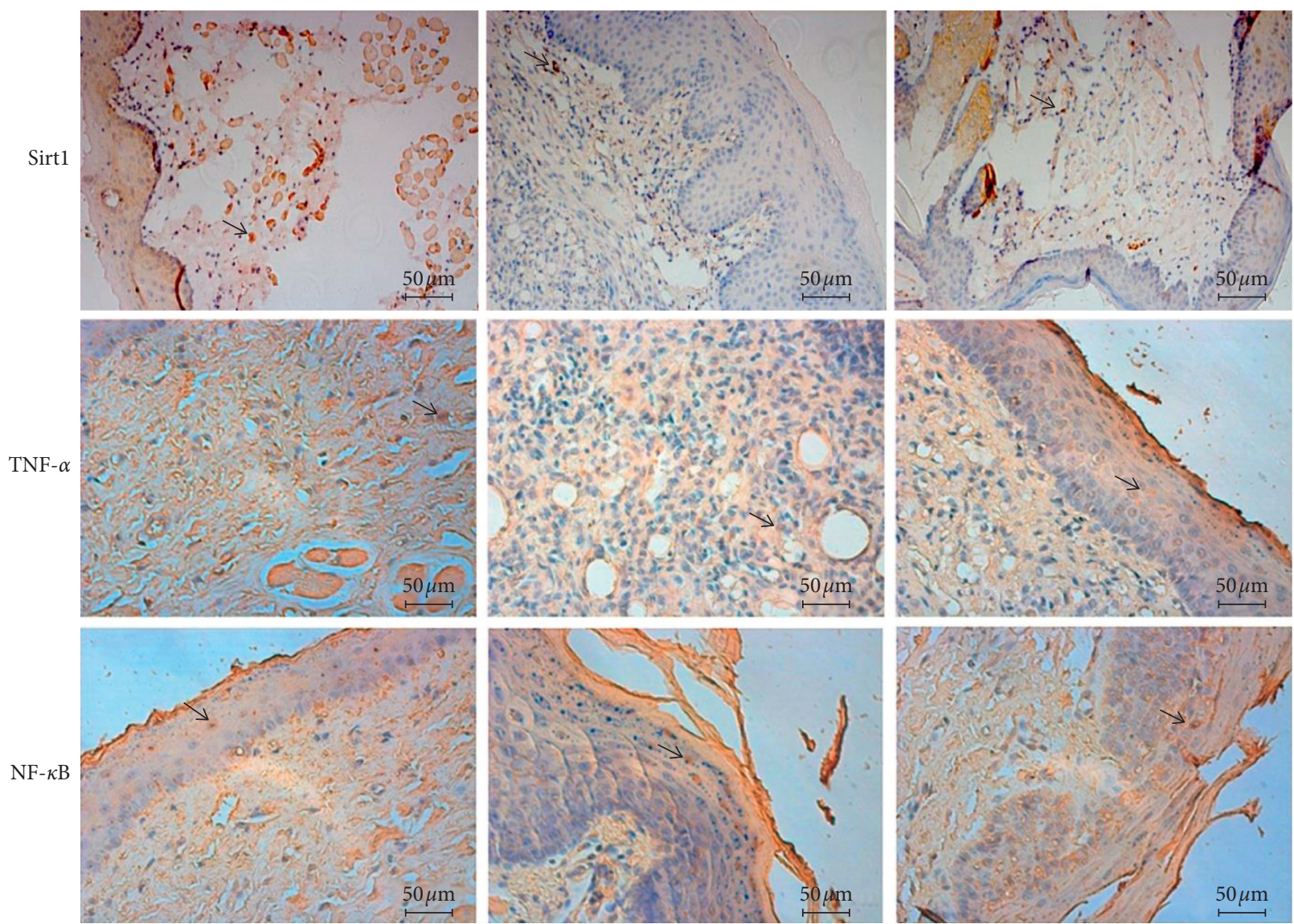

AZC

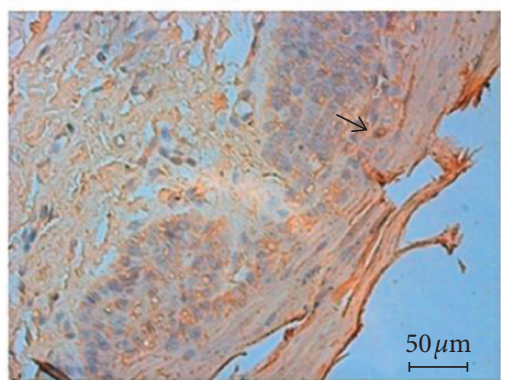

ZBDHD-H

Figure 3: IHC analyses of expression of Sirt1, NF- $\kappa$ B, and TNF- $\alpha$ in oral mucosa. The expressed Sirt1, NF- $\kappa$ B p65, and TNF- $\alpha$ in oral mucosa of Ct, AZC, and ZBDHD-H rats were immunohistochemically (IHC) analyzed and observed under the microscope (x200). The positive cells were pointed out by arrows.
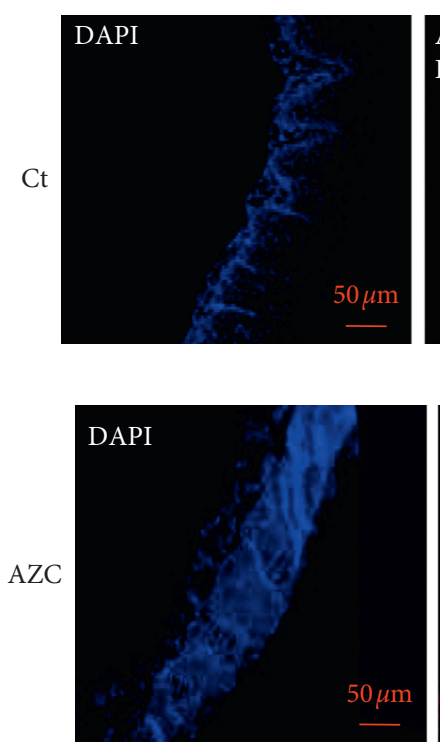
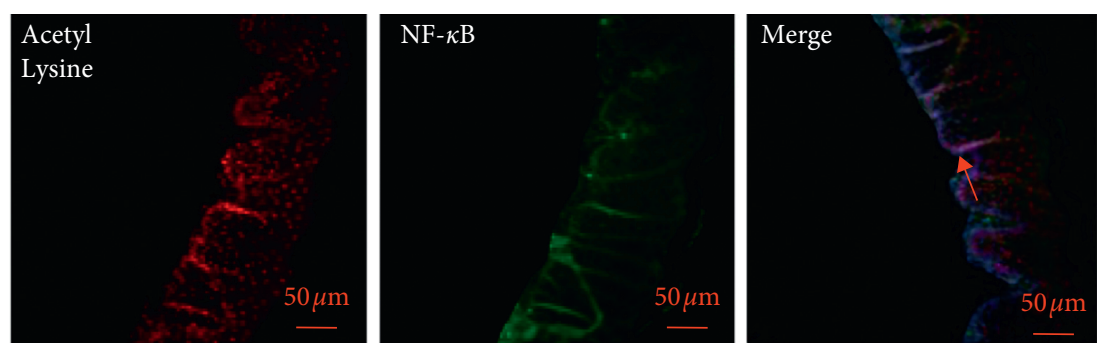

(a)
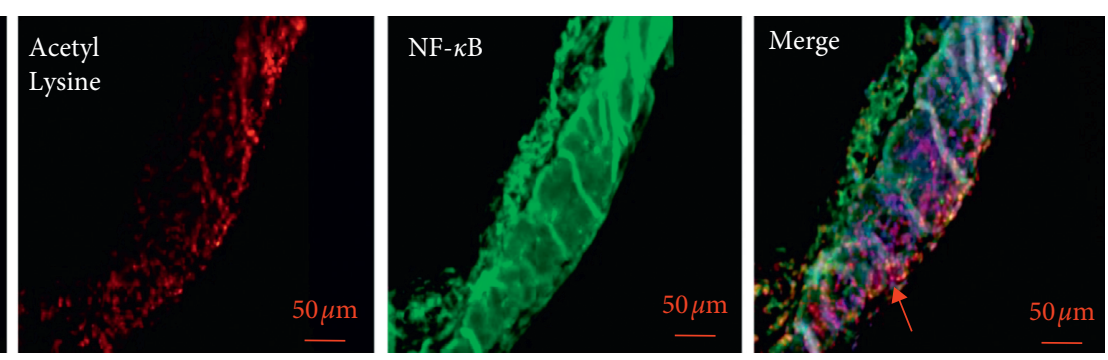

(b)

Figure 4: Continued. 

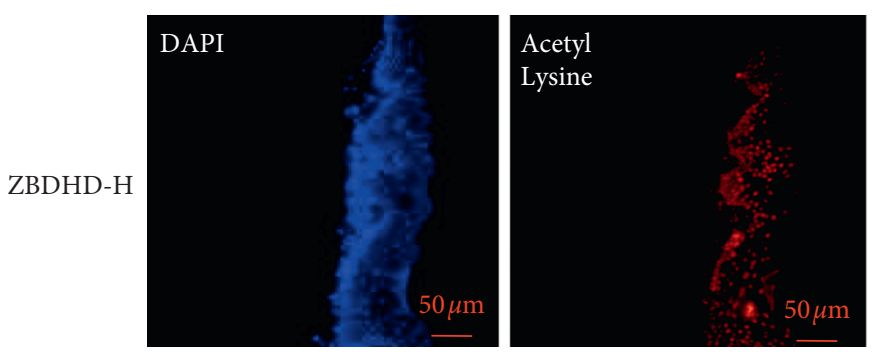

(c)
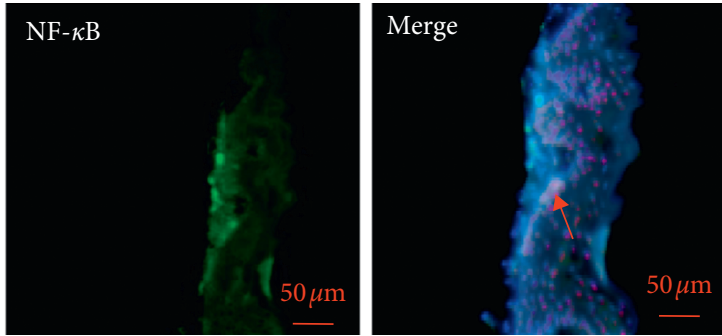

FIgURE 4: IFC demonstration of deacetylation NF- $\kappa \mathrm{B}$ in oral mucosa.

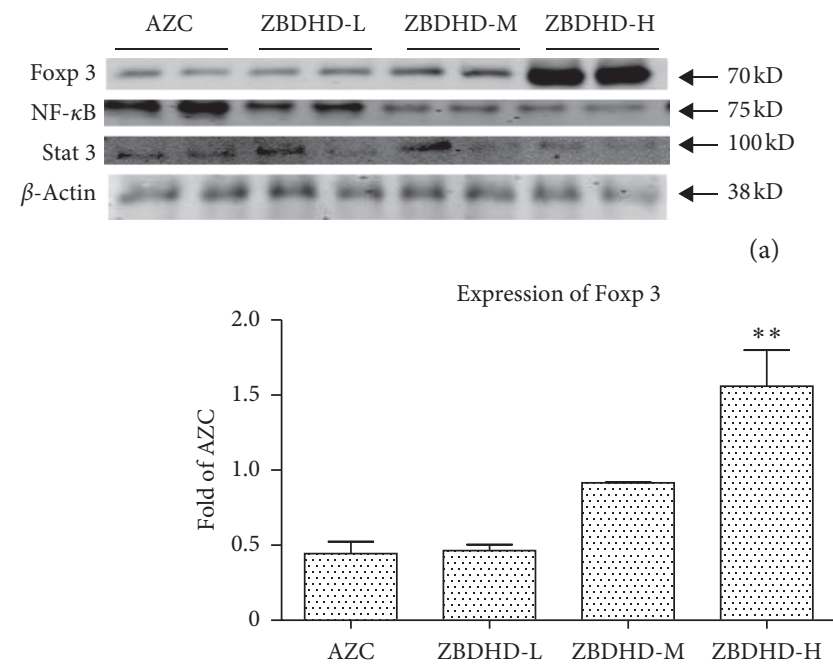

(b)

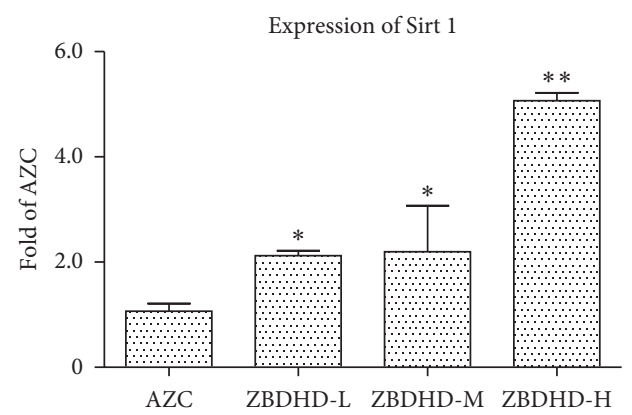

(d)
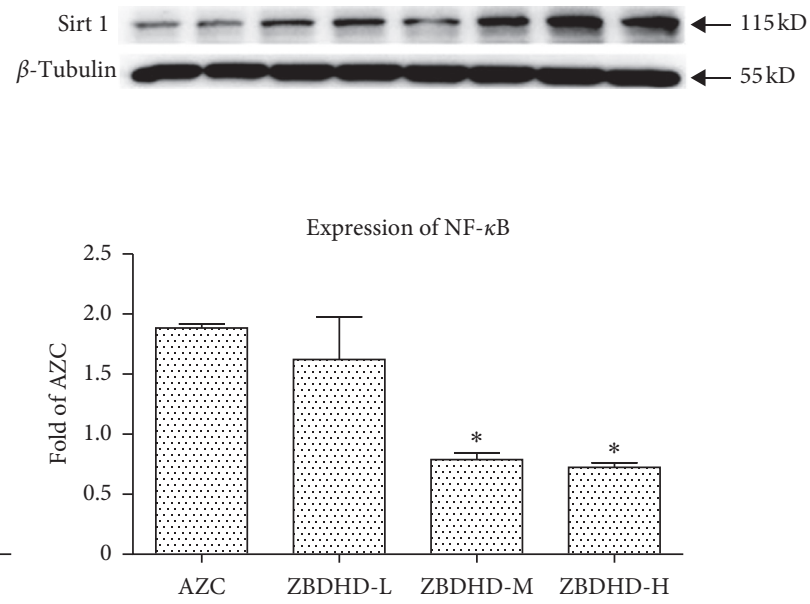

(c)

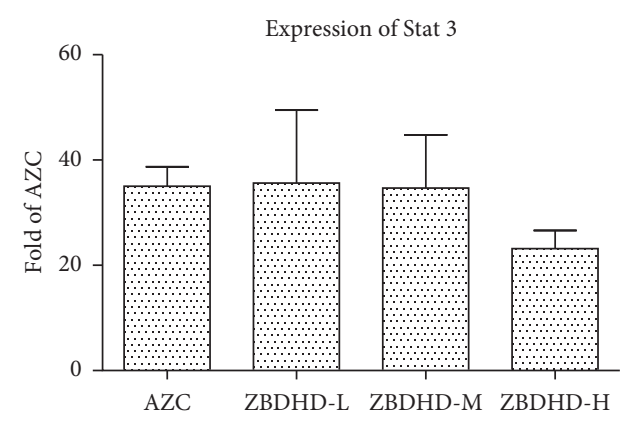

(e)

Figure 5: Expression of Sirt1, Foxp3, NF- $\kappa$ B, and Stat3 in oral mucosa from different group rats. (a) Western blot analyses of Sirt1, Foxp3, NF- $\kappa$ B, and Stat3 in oral mucosa. (b-e) The expression of Sirt1, Foxp3, NF- $\kappa$ B, and Stat3 in ZBDHD-L, ZBDHD-M, and ZBDHD-H versus AZC in oral mucosa; Data are mean $\pm \mathrm{SD}(n=3) .{ }^{*} P<0.05,{ }^{* *} P<0.01$ vs AZC group.

TABLE 3: Docking results of main active compounds in ZBDHD to Sirt1.

\begin{tabular}{|c|c|c|c|c|}
\hline ID & Chemical name & Extraction code & Affinity score & Herb \\
\hline M1458 & Coptisine & $4 Z Z I$ & 10.6 & Phellodendri amurensis Cortex \\
\hline M1454 & Berberine & $4 Z Z I$ & 9.9 & Phellodendri amurensis Cortex \\
\hline M0359 & Sitosterol & 4ZZI & 7.4 & Radix Rehmanniae preparata \\
\hline M0546 & Diosgenin & $4 Z Z J$ & 6.1 & Radix Dioscorea opposita \\
\hline M0211 & Mairin & $4 Z Z J$ & 5.8 & Cortex Paeonia suffruticosa \\
\hline M4489 & Anemarsaponin F_qt & $4 \mathrm{ZZJ}$ & 5.6 & Rhizoma Anemarrhena asphodeloides \\
\hline M0275 & Trametenolic acid & $4 \mathrm{ZZJ}$ & 5.5 & Sclerotium of Poria cocos \\
\hline M0831 & Alisol B monoacetate & $4 \mathrm{ZZJ}$ & 4.8 & Rhizoma Alisma orientale \\
\hline
\end{tabular}




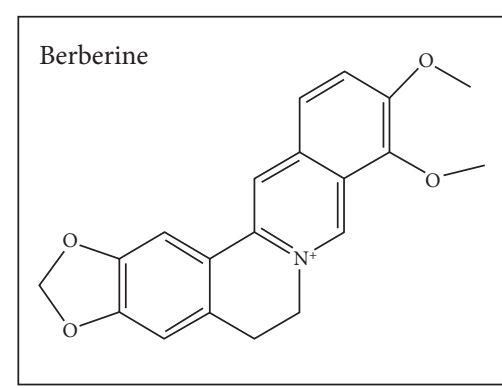

(a)

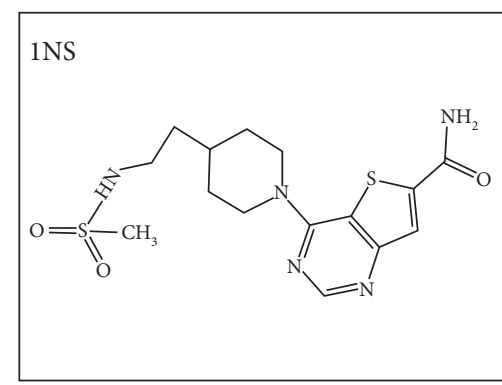

(b)

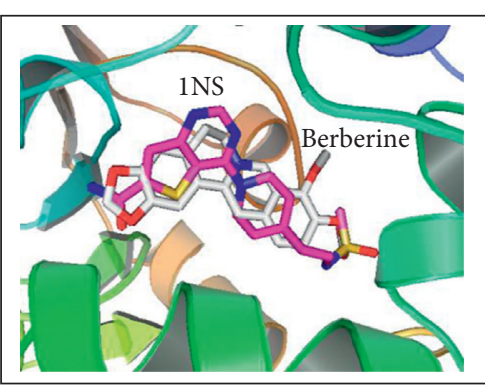

(c)

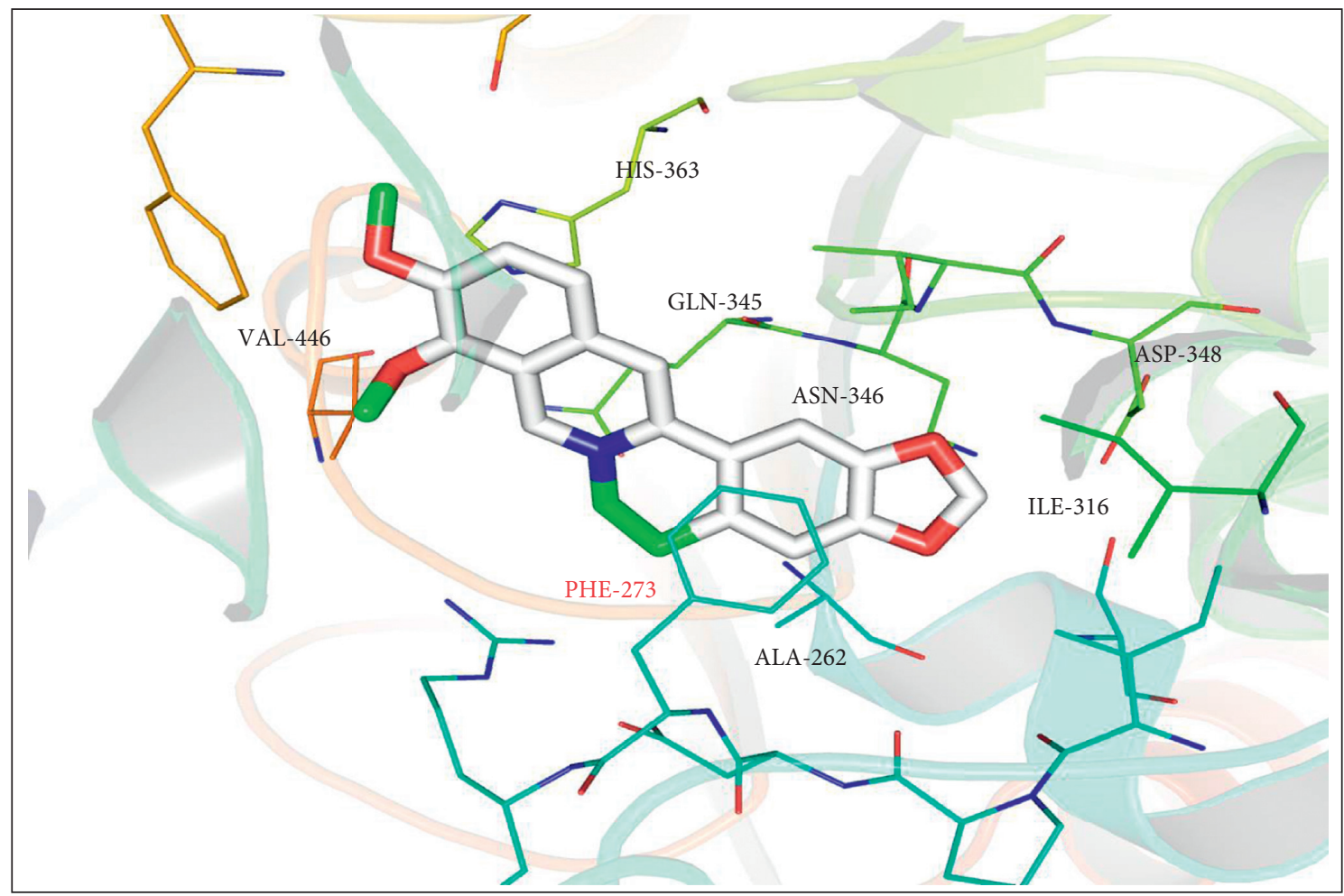

(d)

FIgURE 6: Docking of berberine in active centre of Sirt1. (a) Chemical structure of berberine. (b) Chemical structure of INS. (c) Docking of berberine and INS with Sirt1. (d) Berberine interacts with the amino acid residues in active centre of Sirt1.

disorder of metabolism which could induce oral ulcer [25]. It is validated by clinical trial that ZBDHD treatment could promote the ulcer healing [26] and improve the fitness of recurrent oral ulcer significantly [13].

Our previous research shows the extracts of AZC herbs may induce "fire" syndrome by influencing Treg cells and immunosuppressive cytokines [8]. In this research, AZC combined with immunological method induced immune dysfunction and oral ulcer which was similar to ROU clinical symptoms. Histopathological studies showed the cell inflammation infiltration and mucosal surface damage in model rats (Figures $1(\mathrm{a})$ and $1(\mathrm{c})$ ). The protection capability of ZBDHD was studied in this research. Mucosal surface damage in model and low dose ZBDHD treated rats could be observed under microscope by H\&E staining, but the rats treated with high and middle dose ZBDHD showed a sign of healing which was indicated the absence of damage
(Figure 1). Simultaneously, the difference of metabolites between model group and high dose ZBDHD treatment group was evaluated by metabonomic study which indicated that ZBDHD treatment influenced synthesis and metabolite of some fatty acids, such as phytosphingosine and palmitic amide (Table 2). High dose ZBDHD treatment attenuated AZC induced distractive expression of cytokines, Sirt1, NF- $\kappa$ B, and TNF- $\alpha$ (Figures 3 and 4 ). The results of our previous study also indicated that AZC can stimulate the expression of NF- $\kappa \mathrm{B}$ [13].The expression of cytokines in Sirt1 signaling pathway was quantitatively analyzed by western blot (Figure 5). Sirt1, which belongs to Sirtuins family, is involved in many human physiological processes, such as aging, DNA repair, cell apoptosis [27], and inflammatory response [28]. Sirt1 catalyzes the deacetylation of Lys $310 \mathrm{~s}$ in p65 sigmasubunit of NF- $\kappa \mathrm{B}$ [29], for suppression the regulation 


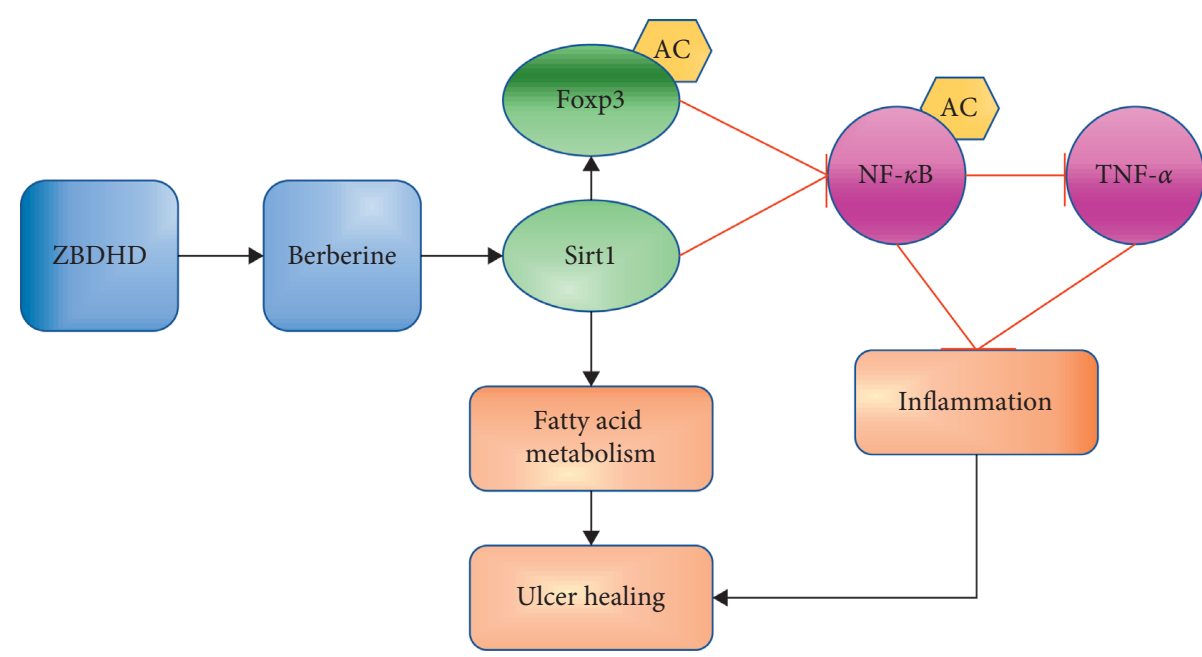

Figure 7: The schematic representation of ZBDHD regulation on ulcer healing and inflammation.

activity of NF- $\kappa \mathrm{B}$ [30]. To predict the phytochemistry and pharmaceutical mechanism of ZBDHD, we used the bioinformatic method to screen TCMSP database (http:// lsp.nwu.edu.cn/tcmsp.php). A total of 78 ingredients present Sirt1 interaction potential. Eight of the 78 show relative high binding affinity with reactive centre of Sirt1 (Table 3). They are coptisine, berberine, sitosterol, diosgenin, mairin, anemarsaponin F_qt, trametenolic acid, and alisol $\mathrm{B}$ monoacetate. They have various pharmaceutical activities. For example, alisol B monoacetate has anti-tumor, anti-allergic, and inhibiting infection from hepatitis B virus [31]. Trametenolic acid can improve cerebral ischemia [32]. Anemarsaponin inhibits inflammation by inhibiting the phosphorylation of NF- $\kappa \mathrm{B}$ p38 [33]. Mairin, also called betulinic acid, has strong effects on anti-HIV, anti-inflammatory, anti-diabetic, and anti-microbial activities [34]. Diosgenin has anti-cancer, anti-diabetic, anti-coagulation, anti-thrombosis, antiinflammatory, anti-viral, and anti-aging properties [35]. Sitosterol has a strong effect on decreasing blood fat and cholesterol [36]. Coptisine and berberine present higher score than others. Both ingredients are the structurally related isoquinoline alkaloids. But the results of previous studies indicated their differential functions. Coptisine, an antioxidant [37], could induce autophagy to exert anti-cancer effects [38]. No references indicated the anti-inflammatory activity of coptisine. In comparison, berberine was reported to have a strong effect on antiinflammatory, previously [39]. As a powerful supplement with many benefits, berberine, one of the eight candidates, showed high binding affinity with the amino acid residues in active centre of Sirt1 (Figure 6). To our knowledge, it is the first time to evaluate the binding site of berberine on Sirt1.

In the present study, we used high dose "hot" herbs potions combined with immunological method to induce ulcer in mouth of model rats. In 14 days, the visible cavity in mucous membrane and decrease of body weight were observed in model rats, in comparison with the other groups. The phenomenon was identical to that in clinic
[40]. Serum metabolomics analysis results showed that the metabolisms of some lipids, such as phytosphingosine, palmitic amide, and MG $(0: 0 / 16: 0 / 0: 0)$, were significantly inhibited by ZBDHD. Phytosphingosine [41], a phospholipid, is a major component of all biological membranes and sphingolipid metabolites. The palmitic amide is one of primary fatty acid amides, which are a kind of signaling molecules [42]. However, the function of palmitic amide is less known. MG $(0: 0 / 16: 0 / 0: 0)$ is a monoacylglyceride, consisting of a fatty acid chain covalently bonded to a glycerol molecule through an ester linkage. It is an intermediate in glycerophospholipid metabolism process [43]. On the contrary, ZBDHD treatment upregulated the serum concentrations of $\alpha$-linolenic acid and LysoPC (20:4 (5Z, $8 Z, 11 Z, 14 Z))$, LysoPC (20:3 (5Z, 8Z, 11Z), and LysoPE (0: $0 / 20: 0)$. Both LysoPC $(20: 4(5 Z, 8 Z, 11 Z, 14 Z))$ and LysoPC (20:3 (5Z, 8Z, 11Z)) are lysophosphatidylchoine. LysoPE $(0: 0 / 20: 0)$ are lysophosphatidylethanolamine. They are structural compounds in cell membrane at a low density. Thus, they are biomarkers of cell proliferation [44]. In addition, LysoPC and LysoPE are both post-injury marker metabolites [45]. LysoPC was a strong chemoattractant of T-lymphocytes, which can promote antibody formation and macrophages stimulus [46]. LysoPE is mobilizer of intracellular $\mathrm{Ca}^{2+}$ in some cell types $[47,48]$. Calcium is one of the initial triggers in immune response for wound healing [49]. $\alpha$-Linolenic acid served as substrate for unsaturated fatty acid synthesis, which showed anti-inflammatory effects [50]. Therefore, the results of metabolomics analysis indicated that the ZBDHD can reduce inflammation and promote the ulcer healing.

It had been reported that Sirt1, the silent information adjustment factor 2 related enzyme I, could suppress fatty acid metabolism [51]. In addition, Sirt1 can catalyze acetylation of transcription factor Forkhead Box P3 (Foxp3), an inhibitor of NF- $\kappa B$ [52]. NF- $\kappa \mathrm{B}$ is an important pro-inflammatory factor and could influence the expression of inflammatory factors, such as TNF- $\alpha$, IL- $1 \beta$, and IL-8 [53]. Therefore, we hypothesized that Sirt1 could be one of the target factors of ZBDHD treatment. 


\section{Conclusion}

In present study, we demonstrated that ZBDHD treatment upregulated the expression of Sirt1 and attenuated the overexpression of TNF- $\alpha$. These results confirmed that Sirt1 may be the main target of $\mathrm{ZBDHD}$ to reduce the mucosa inflammation. ZBDHD could improve the symptoms of oral ulcer in model rats. And the capability mechanism may be related to Sirt1-NF- $\kappa$ B pathway, immune regulation, and metabolism regulation. And the berberine, one of the constituents in ZBDHD, is a key regulator of Sirt1 (Figure 7).

\section{Data Availability}

All data used to support the findings of this study are included within the article, and these data can also be made accessible on website https://figshare.com/s/3ba0eaf51b3c2c78bc7b.

\section{Conflicts of Interest}

The authors declare that they have no conflicts of interest.

\section{Authors' Contributions}

All the authors provided critical feedback and contributed to research designation, data collection, and analysis, respectively. In detail, Ms Yajie Shao and Shanshan Lei performed the experiments and collected data. Associate professor Bin Ding analyzed the data and drafted this manuscript. Ms Jinjun Ji was responsible for lab management and helped with laboratory procedure. Dr Yu Dong was responsible for serum metabolomics analysis. Professor YS Fan designed the animal model preparation and financially supported this study. Mr Weihong Ge helped in modifying and reediting this manuscript. Professor L Xu contributed to the conception of the whole work and financially supported this work.

\section{Acknowledgments}

The authors acknowledge the financial supports of National Basic Research Program of China (973 Program), Grant No. $2014 \mathrm{CB} 543000$ and Natural Science Foundation of China (81673857).

\section{Supplementary Materials}

Table S1: related pathways of differences metabolites. Table S2: screening out 78 kinds of active compounds in Zhibaidihuang decoction. (Supplementary Materials)

\section{References}

[1] P. P. Toche, L. J. Salinas, M. M. Guzman, A. S. Alejandro, and J. A. Nicole, "Recurrent oral ulcer: clinical characteristic and differential diagnosis," Revista Chilena de Infectologí, vol. 24, no. 3, pp. 215-219, 2007.

[2] A. S. Ahmed, K. Vesa-Petteri, S. Abdelhakim et al., "IL-17C and its receptor IL-17RA/IL-17RE identify human oral epithelial cell as an inflammatory cell in recurrent aphthous ulcer," Journal of Oral Pathology \& Medicine, vol. 43, no. 2, pp. 117-124, 2014.

[3] N. Lewkowicz, P. Lewkowicz, K. Dzitko et al., "Dysfunction of $\mathrm{CD} 4{ }^{+} \mathrm{CD} 25^{\text {high }} \mathrm{T}$ regulatory cells in patients with recurrent aphthous stomatitis," Journal of Oral Pathology \& Medicine, vol. 37, no. 8, pp. 454-461, 2010.

[4] Y. H. Zou, J. Yang, and C. H. Chen, "A study on correlation of serum TNF- $\alpha$, IL-2, 6 concentration and immune function in patients with recurrent oral ulcers," Journal of Hainan Medical University, vol. 2015, no. 9, pp. 161-163, 2015.

[5] X. M. Yang, X. H. Wang, L. F. Chen, and X. Q. Wang, "Effects of dihydromyricetin on tumor necrosis factor and NF-kappaB p65 of RAU rats," China Journal of Chinese Materia Medica, vol. 37, no. 17, pp. 2612-2617, 2012.

[6] P. Chen, H. Yao, W. Su et al., "Sleep deprivation worsened oral ulcers and delayed healing process in an experimental rat model," Life Science, vol. 232, no. 1, Article ID 116594, 2019.

[7] M. Q. He, X. Bao, and C. P. Wen, "Clinical features of the fire-heat symptom," China Journal of Traditional Chinese Medicine \& Pharmacy, vol. 28, no. 3, pp. 791-792, 2013.

[8] D. H. Wu, L. Xu, G. Q. Xie, Y. S. Fan, and J. Zhou, "The pungent and hot Chinese herbs cause heat syndrome in rats by affecting the regulatory T cells," Evidence-based Complementary and Alternative Medicine, vol. 2019, Article ID 9824906, 9 pages, 2019.

[9] J. J. Ji, Y. F. Jiao, X. Y. Chen et al., "Study on the mechanism of oral ulcer induced by excessive shang-huo in rats induced by ganjiang fuzi rougui decoction," Journal of Zhejiang University of Traditional Chinese Medicine, vol. 42, no. 10, pp. 775-781, 2018.

[10] B. Han and S. Wang, "Research on molecular mechanism of "yin asthenia with internal heat" in asthenia hot syndrome rats by gene chip technology," Journal of Sichuan of Traditional Chinese Medicine, vol. 31, no. 8, pp. 37-40, 2013.

[11] C. Q. Chen and Y. F. Chen, "Efficacy observation of recurrent oral ulceration with combination of zhibai dihuang pills and compound danshen tablets," China Pharmacy, vol. 26, no. 26, pp. 3693-3695, 2015.

[12] D. F. Wang, “Therapeutic effect of chronic periodontitis with zhibai dihuang pills combined with periocline and its effect on inflammatory factor in gingival crevicular fluid," Chinese Journal of Experimental Traditional Medical Formulae, vol. 18, no. 12, pp. 273-275, 2012.

[13] P. P. Wu, B. Ding, L. Ye et al., "Zhibaidihuang decoction ameliorates cell oxidative stress by regulating the Keap1-Nrf2ARE signalling pathway," Evidence-Based Complementary and Alternative Medicine, vol. 2020, Article ID 9294605, 11 pages, 2020.

[14] Y.-P. Chen, C.-W. Tsai, C.-Y. Shen et al., "Palmitic acid interferes with energy metabolism balance by adversely switching the SIRT1-CD36-fatty acid pathway to the PKC zeta-GLUT4-glucose pathway in cardiomyoblasts," The Journal of Nutritional Biochemistry, vol. 31, pp. 137-149, 2016.

[15] X. Meng, J. Tan, M. Li, S. Song, Y. Miao, and Q. Zhang, "Sirt1: role under the condition of ischemia/hypoxia," Cellular and Molecular Neurobiology, vol. 37, no. 1, pp. 17-28, 2017.

[16] K. Qin, C. Han, H. Zhang, T. Li, N. Li, and X. Cao, "NAD + dependent deacetylase Sirtuin 5 rescues the innate inflammatory response of endotoxin tolerant macrophages by promoting acetylation of p65," Journal of Autoimmunity, vol. 81, pp. 120-129, 2017.

[17] K. L. Mendes, D. D. F. Lelis, and S. H. S. Santos, "Nuclear sirtuins and inflammatory signaling pathways," Cytokine \& Growth Factor Reviews, vol. 38, pp. 98-105, 2017. 
[18] L. Xu, J.-J. Ji, W. Le et al., "The STAT3 HIES mutation is a gain-of-function mutation that activates genes via AGG-element carrying promoters," Nucleic Acids Research, vol. 43, no. 18, pp. 8898-8912, 2015.

[19] J. L. Ru, P. Li, J. Wang et al., "TCMSP: a database of systems pharmacology for drug discovery from herbal medicines," Journal of Cheminformatics, vol. 6, no. 1, pp. 1-6, 2014.

[20] G. M. Morris, R. Huey, and A. J. Olson, "Using autodock for ligand-receptor docking," Current Protocols in Bioinformatics, vol. 8, no. 8, pp. 775-781, 2008.

[21] R. Huo, K. Q. Liu, Y. Q. Li et al., "Efficacy of the zhibai dihuang decoction on type 2 diabetes of the yinxu huowang type," Clinical Journal of Chinese Medicine, vol. 11, no. 16, pp. 129-131, 2019.

[22] Y. Q. Zhao, Q. S. Li, M. H. Xiang et al., "Comparison of internal and external therapies for the treatment of recurrent aphthous ulcer and study on the rule of drug use of the main "syndrome elements" and "syndrome type," China Journal of Traditional Chinese Medicine and Pharmacy, vol. 33, no. 5, pp. 2174-2183, 2018.

[23] L. Preeti, K. Magesh, K. Rajkumar, and R. Karthik, "Recurrent aphthous stomatitis," Journal of Oral and Maxillofacial Pathology, vol. 15, no. 3, pp. 252-256, 2011.

[24] S. K. Saikaly, T. S. Saikaly, and L. E. Saikaly, "Recurrent aphthous ulceration: a review of potential causes and novel treatments," Journal of Dermatological Treatment, vol. 29, no. 6, pp. 542-552, 2018.

[25] N. Sukhitashvili, I. Imnadze, G. Tabaghua, Q. Gogilashvili, and I. Amiranashvili, "Characterization of oral ulcer and pathological scar in nude mice model," Georgian Medical News, vol. 205, pp. 82-87, 2012.

[26] L. Zhang, "Zhibai dihuang pill in the treatment of 39 cases of recurrent oral ulcer," Clinical Journal of Chinese Medicine, vol. 8, no. 27, pp. 101-102, 2016.

[27] E. S. Hwang and S. B. Song, "Nicotinamide is an inhibitor of SIRT1 in vitro, but can be a stimulator in cells," Cellular and Molecular Life Sciences, vol. 74, no. 18, pp. 3347-3362, 2017.

[28] L. Yang, J. Zhang, C. Yan et al., "SIRT1 regulates CD40 expression induced by TNF- $\alpha$ via NF- $\kappa$ B pathway in endothelial cells," Cellular Physiology and Biochemistry, vol. 30, no. 5, pp. 1287-1298, 2012.

[29] X. L. Cui, Q. Chen, Z. Dong et al., "Inactivation of Sirt1 in mouse livers protects against endotoxemic liver injury by acetylating and activating NF- $\kappa \mathrm{B}$," Cell Death \& Disease, vol. 7, no. 10, Article ID e2403, 2016.

[30] H. Y. Yang, W. Zhang, H. Pan et al., "SIRT1 activators suppress inflammatory responses through promotion of p65 deacetylation and inhibition of NF- $\kappa \mathrm{B}$ activity," PLoS One, vol. 7, no. 9, Article ID e46364, 2012.

[31] T. Chen and B. Liu, "Advances in the study of alisol B acetate extracted from alismatis rhizoma," Bulletin of Science and Technology, vol. 2008, no. 4, pp. 485-488, 2008.

[32] J. Wang, A. Wang, H. He et al., "Trametenolic acid B protects against cerebral ischemia and reperfusion injury through modulation of microRNA-10a and PI3K/Akt/mTOR signaling pathways," Biomedicine \& Pharmacotherapy, vol. 112, Article ID 108692, 2019.

[33] J.-Y. Kim, J.-S. Shin, J. H. Ryu et al., “Anti-inflammatory effect of anemarsaponin B isolated from the rhizomes of anemarrhena asphodeloides in LPS-induced RAW 264.7 macrophages is mediated by negative regulation of the nuclear factor- $\kappa \mathrm{B}$ and $\mathrm{p} 38$ pathways," Food and Chemical Toxicology, vol. 47, no. 7, pp. 1610-1617, 2009.
[34] C. A. Barreto, B. Ronan, M. Á. Castro, and J. M. David, "Chemical strategies towards the synthesis of betulinic acid and its more potent antiprotozoal analogues," Molecules, vol. 26, no. 4, pp. 1081, 2021.

[35] R. Nazir, V. Kumar, S. Gupta, D. K. Pandey, and A. Dey, "Biotechnological strategies for the sustainable production of diosgenin from dioscorea spp," Applied Microbiology and Biotechnology, vol. 105, no. 2, pp. 569-585, 2021.

[36] W. L. Liu, Y. Ji, and A. X. Huang, "Research and development progress of $\beta$-sitosterol," Farm Products Processing, vol. 1, pp. 77-79, 2019.

[37] H.-G. Jo, C. Park, H. Lee et al., "Inhibition of oxidative stress induced-cytotoxicity by coptisine in V79-4 Chinese hamster lung fibroblasts through the induction of Nrf-2 mediated HO1 expression," Genes \& Genomics, vol. 43, no. 1, pp. 17-31, 2021.

[38] S. Y. Kim, H. Hwangbo, M. Y. Kim et al., "Coptisine induces autophagic cell death through down-regulation of PI3K/Akt/ mTOR signaling pathway and up-regulation of ROS-mediated mitochondrial dysfunction in hepatocellular carcinoma Hep3B cells," Archives of Biochemistry and Biophysics, vol. 15, no. 697, Article ID 108688, 2021.

[39] K. Zou, Z. Li, Y. Zhang et al., "Advances in the study of berberine and its derivatives: a focus on anti-inflammatory and anti-tumor effects in the digestive system," Acta Pharmacologica Sinica, vol. 38, no. 2, pp. 157-167, 2017.

[40] A. Anura, "Traumatic oral mucosal lesions: a mini review and clinical update," Oral Health and Dental Management, vol. 13, no. 2, pp. 254-259, 2014.

[41] N. Kondo, Y. Ohno, M. Yamagata et al., "Identification of the phytosphingosine metabolic pathway leading to odd-numbered fatty acids," Nature Communications, vol. 5, no. 1, pp. 270-278, 2014.

[42] E. K. Farrell, Y. Chen, M. Barazanji, K. A. Jeffries, F. Cameroamortegui, and D. J. Merkler, "Primary fatty acid amide metabolism: conversion of fatty acids and an ethanolamine in N18TG2 and SCP cells," Journal of Lipid Research, vol. 53, no. 2, pp. 247-256, 2012.

[43] P. M. Zygmunt, A. Ermund, P. Movahed et al., "Monoacylglycerols activate TRPV1-a link between phospholipase C and TRPV1," PLoS One, vol. 8, no. 12, Article ID e81618, 2013.

[44] H. L. Zhou, J. H. Yao, and S. Yu, "The effect of the LysoPCinduced endothelial cell conditioned medium on proliferating cell nuclear antigen expression of the calf thoracic aorta smooth muscle cells," Journal of Huazhong University of Science and Technology, vol. 22, no. 1, pp. 28-30, 2002.

[45] J. O. Ojo, M. Algamal, P. Leary et al., "Disruption in brain phospholipid content in a humanized tau transgenic model following repetitive mild traumatic brain injury," Frontiers in Neuroscience, vol. 12, p. 893, 2018.

[46] X. Ding, J. Hu, J. Li et al., "Metabolomics analysis of collageninduced arthritis in rats and interventional effects of oral tolerance," Analytical Biochemistry, vol. 458, pp. 49-57, 2014.

[47] S.-J. Park, K.-P. Lee, and D.-S. Im, "Action and signaling of lysophosphatidylethanolamine in MDA-MB-231 breast cancer cells," Biomolecules \& Therapeutics, vol. 22, no. 2, pp. 129-135, 2014.

[48] J.-M. Lee, S.-J. Park, and D.-S. Im, "Calcium signaling of lysophosphatidylethanolamine through LPA1in human $\mathrm{SH}$ SY5Y neuroblastoma cells," Biomolecules \& Therapeutics, vol. 25, no. 2, pp. 194-201, 2017.

[49] W. Razzell, I. R. Evans, P. Martin, and W. Wood, "Calcium flashes orchestrate the wound inflammatory response through 
DUOX activation and hydrogen peroxide release," Current Biology, vol. 23, no. 5, pp. 424-429, 2013.

[50] S. D. Pauls, L. A. Rodway, T. Winter, C. G. Taylor, P. Zahradka, and H. M. Aukema, "Anti-inflammatory effects of $\alpha$-linolenic acid in M1-like macrophages are associated with enhanced production of oxylipins from $\alpha$-linolenic and linoleic acid," The Journal of Nutritional Biochemistry, vol. 57, pp. 121-129, 2018.

[51] B. R. Zhou, J. A. Zhang, Q. Zhang et al., "Palmitic acid induces production of proinflammatory cytokines interleukin-6, interleukin- $1 \beta$, and tumor necrosis factor- $\alpha$ via a NF- $\kappa \mathrm{B}$ dependent mechanism in HaCaT keratinocytes," Mediators of Inflammation, vol. 2013, Article ID 530429, 11 pages, 2013.

[52] X. Yang, Y. Lun, H. Jiang et al., "SIRT1-Regulated abnormal acetylation of FOXP3 induces regulatory T-cell function defect in hashimoto's thyroiditis," Thyroid, vol. 28, no. 2, pp. 246-256, 2018.

[53] L. Luo, Y. Gao, C. Yang et al., "Halofuginone attenuates intervertebral discs degeneration by suppressing collagen I production and inactivating TGF $\beta$ and NF-kB pathway," Biomedicine \& Pharmacotherapy, vol. 101, pp. 745-753, 2018. 